\title{
Overexpression of peroxiredoxin-3 and -5 is a potential biomarker for prognosis in endometrial cancer
}

\author{
JUNG MI BYUN ${ }^{1,2 *}$, SU SUN KIM ${ }^{1 *}$, KI TAE KIM ${ }^{1,2}$, MI SEON KANG $^{3}$, DAE HOON JEONG ${ }^{1,2}$, \\ DAE SIM LEE ${ }^{1,2}$, EUN JUNG JUNG ${ }^{1}$, YOUNG NAM KIM ${ }^{1,2}$, JIN HAN $^{4}$, \\ IN SUNG SONG ${ }^{4}, \mathrm{KYOUN}^{\mathrm{BOK}} \mathrm{LEE}^{1,2}$ and MOON SU SUNG ${ }^{1,2}$ \\ ${ }^{1}$ Department of Obstetrics and Gynecology; ${ }^{2}$ Paik Institute for Clinical Research; Departments of ${ }^{3}$ Pathology and \\ ${ }^{4}$ Physiology, Inje University Busan Paik Hospital, Busan 614-735, Republic of Korea
}

Received August 16,2016; Accepted August 24, 2017

DOI: $10.3892 / 01.2018 .7909$

\begin{abstract}
Endometrial cancer is the sixth most common cancer in women worldwide. Peroxiredoxins (PRDXs) are antioxidant enzymes that serve important roles in cell differentiation, proliferation, and apoptosis. In the present study, the potential associations between PRDX expression and endometrial cancer were investigated. The expression levels of various PRDX mRNAs were detected by semi-quantitative reverse transcription polymerase chain reaction (RT-PCR) in endometrial cancer tissues $(n=26)$ and normal endometrial tissues $(n=10)$. Additionally, the expression of PRDX isoforms was immunohistochemically examined in endometrial cancer tissues and adjacent normal endometrial tissues from 42 patients. Finally, the associations between high PRDX expression levels and clinicopathological features were examined in patients with endometrial cancer. Analysis of PRDX expression in endometrial cancer tissues and normal endometrial tissues by semi-quantitative RT-PCR showed that all PRDX isoforms had increased expression in the endometrial cancer tissues compared with that in the normal endometrium, and the differences in the expression levels of $P R D X 1$ and $P R D X 3$ between cancer and normal tissues were statistically significant $(\mathrm{P}=0.0015$ and $\mathrm{P}=0.0134$, respectively). Additionally, analysis of PRDX expression in
\end{abstract}

Correspondence to: Professor Ki Tae Kim, Department of Obstetrics and Gynecology, Inje University Busan Paik Hospital, 75 Bokji-ro, Busanjin-gu, Busan 614-735, Republic of Korea

E-mail: hellojungmi@hanmail.net

Professor Mi Seon Kang, Department of Pathology, Inje University Busan Paik Hospital, 75 Bokji-ro, Busanjin-gu Busan 614-735, Republic of Korea

E-mail: pathmsk@hanmail.net

*Contributed equally

Abbreviations: PRDX, peroxiredoxin; hB2MF, human 32 -microglobulin; LVSI, lymphovascular space invasion

Key words: endometrial cancer, prognostic marker, peroxiredoxin endometrial cancer and paired normal endometrial tissues by immunohistochemistry showed strong cytoplasmic staining of PRDX3 and PRDX5 in cancer tissues, with high PRDX3 (25/42, 59.5\%) and PRDX5 (32/42, 76.2\%) appearing more frequently in endometrial cancer than in normal endometrial tissues $(\mathrm{P}=0.0001$ and $\mathrm{P}=0.0023$, respectively). Furthermore, high expression of PRDX5 was associated with advanced-stage endometrial cancer $(\mathrm{P}=0.0399)$. Although the 5-year survival rate was marginally higher in patients with low expression of PRDX3 and PRDX5, this result was not statistically significant. In summary, PRDX3 and PRDX5 are highly expressed in endometrial cancer and could be associated with advanced stage and poor prognosis. Therefore, these proteins may potentially be used as prognostic markers for endometrial cancer.

\section{Introduction}

Endometrial cancer is the sixth most common cancer in women worldwide; approximately 320,000 new cases were diagnosed in 2012 (1). The incidence rate and number of mortalities from endometrial cancer are increasing, despite improvements in overall survival rates (2). Overall survival rates for endometrial cancer are good, and the 5-year survival rate for disease confined to the uterus is as high as $96 \%$ (3). In the majority of cases, patients with endometrial cancer exhibit symptoms that include abnormal vaginal bleeding or discharge, and $290 \%$ of women diagnosed with endometrial cancer have abnormal uterine bleeding (4). Therefore, in pre- and post-menopausal women exhibiting abnormal uterine bleeding, transvaginal ultrasonography and endometrial biopsy should be performed for the potential early diagnosis of endometrial cancer (5). Early detection of endometrial cancer improves prognosis; therefore, improved diagnostic methods are critical for patients with symptoms of endometrial cancer, and screening methods for the detection of early-stage cancer prior to the onset of symptoms are expected to achieve meaningful improvements in overall survival. However, there are currently no routine, effective screening tests for endometrial cancer (6).

Tumor markers may be secreted by tumors at levels in excess of those secreted by normal tissues and cells (7). Such tumor markers can include unique extracellular matrix or cell adhesion molecules, growth factors, receptors, 
cytokines, or products of abnormal metabolic processes. In addition, antibodies produced by the body against tumor markers may themselves be used as markers. Tumor markers can be used as indicators of diagnosis, prognosis, and can informative for clinical management and follow-up (7). Numerous investigations have assessed different biological variables in tissue and serum samples from patients with endometrial cancer, in order to detect possible biomarkers that could be predictive of clinical outcomes. For example, Gadducci et al (8) reported the ability of different tissue and serum biomarkers, including p53, the PENT-PIK3-mTOR signaling pathway, MSI, $\beta$-Catenin, the Ras-MAPK-ERK signaling pathway and VEGF in tissue and CA 125, CA15-3, YKL-40 VEGF and HE-4 in serum, to predict clinical outcomes in patients with endometrioid-type endometrial carcinoma. Elevated serum CA125 levels have been detected in $11-43 \%$ of patients with endometrial cancer $(9,10)$. Investigators have shown that elevated CA125 levels are able to predict extrauterine lesions, large tumor size, invasion of the lymphovascular space and deep myometrium, involvement of the cervix and adnexa, positive cytology, lymph node metastasis, and the requirement for adjuvant treatment (11). However, in patients with pure endometrioid-type endometrial cancer who have undergone adjuvant therapy and in patients with serous papillary carcinoma, the role of serum CA125 is controversial (12). Additionally, CA125 has limited utility for monitoring the response to chemotherapy and may not predict recurrence in the absence of other clinical signs (13). Therefore, additional studies are required to identify novel tumor markers for more accurate detection and management of endometrial cancers.

Peroxiredoxins (PRDXs), which were first discovered $\sim 25$ years ago (14), are a family of 22-to $27-\mathrm{kDa}$, non-selenium-dependent glutathione peroxidases that destroy peroxides, organic hydroperoxides and peroxynitrite (15). The PRDX gene family (16) includes six isoforms in mammals; these isoforms can be classified into three subclasses: Typical 2-cysteine PRDXs (PRDXs 1-4), atypical 2-cysteine PRDX (PRDX5), and atypical 1-cysteine PRDX (PRDX6) (17). PRDXs are associated with cell proliferation, apoptosis, differentiation, and gene expression in vitro (18). Notably, high expression levels of PRDXs are associated with increased resistance to radiation and certain chemotherapeutics, whereas PRDX deficiency can sensitize cells to chemotherapy and apoptosis (19).

In addition, oxidative metabolism of estrogen and the subsequent formation of reactive oxygen species (ROS) are important estrogen-related carcinogenic mechanisms (20). Felty et al (21) suggested that physiological estrogen concentrations could induce significant oxidative stress in vitro and that estrogen-induced ROS formation occurs in the mitochondria. For example, in estrogen receptor (ER)-positive breast cancer, which is a major estrogen-dependent cancer, estrogens are important cellular ROS inducers (22). Additionally, published data suggest that PRDX1, PRDX3, PRDX4 and PRDX5 expression levels are associated with prognosis in patients with breast cancer (23-25). Similarly, endometrial cancer is an estrogen-dependent malignancy; therefore, certain PRDXs may be associated with the prognosis or clinicopathological characteristics of endometrial cancer.
Accordingly, in the present study, the expression levels of various PRDXs were evaluated in endometrial cancer tissues, and the relationship between PRDX expression and prognosis in patients with endometrial cancer was investigated.

\section{Materials and methods}

Tissues. Fresh tissue specimens were collected from the endometrial tissues of 70 patients who underwent hysterectomy at Inje University Busan Paik Hospital (Busan, Korea) between January 2008 and December 2010. Using a retrospective chart review, a database was established containing information regarding prognostic factors, such as age, body mass index, histopathological factors, stage, recurrence, and survival. Tissue specimens were classified into two groups: Normal endometrium and endometrial cancer. 42 patients with endometrial cancer received full staging surgery, including total hysterectomy, bilateral salpingo-oophorectomy, omentectomy, bilateral pelvic lymphadenectomy and para-aortic lymphadenectomy. Endometrial carcinomas are graded by their architecture and revised International Federation of Gynecology and Obstetrics (FIGO) classification (26) is used for their staging. Normal endometrial tissues were obtained from hysterectomies performed for benign uterine disease, including uterine myoma, adenomyosis, or uterine prolapse.

This study was performed following the acquisition of informed consent from all patients and approval from the Institutional Review Board of Busan Paik Hospital.

RNA isolation and cDNA synthesis. Total RNA was isolated from tissue samples using TRIzol reagent (Invitrogen; Thermo Fisher Scientific, Inc., Waltham, MA, USA) according to the manufacturer's protocol.cDNA was synthesized from $1 \mu \mathrm{g}$ total RNA using a TOPscript ${ }^{\mathrm{TM}}$ cDNA Synthesis Kit (Enzynomics, Daejeon, Korea). The reverse transcription (RT) reaction was performed at $42^{\circ} \mathrm{C}$ for $60 \mathrm{~min}$ in a reaction mixture containing 10X TOPscript ${ }^{\mathrm{TM}}$ RT reaction MIX (dT18) and TOPscript ${ }^{\mathrm{TM}}$ Reverse Transcriptase $^{\mathrm{R}+}(200 \mathrm{U} / \mu \mathrm{l})$ in a total volume of $20 \mu \mathrm{l}$.

Semi-quantitative RT-polymerase chain reaction (PCR). The RT product $(1 \mu \mathrm{l})$ was used for PCR. Each reaction contained $10 \mathrm{pM}$ of each primer, $1.5 \mathrm{mM} \mathrm{MgCl} 2,250 \mu \mathrm{M}$ dNTPs, $10 \mathrm{mM}$ Tris- $\mathrm{HCl}$ (pH 9.0), $30 \mathrm{mM} \mathrm{KCl}$, and 1 unit Top DNA polymerase (AccuPower ${ }^{\circledR}$ PCR PreMix; cat. no. K-2012; Bioneer Corporation, Daejeon, Korea). The primers utilized are presented in Table I. Reactions were performed on a T100 Thermal Cycler (Bio-Rad, Hercules, CA, USA). Amplification was conducted with the following thermal cycling conditions: $5 \mathrm{~min}$ at $95^{\circ} \mathrm{C} ; 35$ cycles of amplification consisting of $30 \mathrm{sec}$ at $95^{\circ} \mathrm{C}, 40 \mathrm{sec}$ at $55^{\circ} \mathrm{C}$, and $30 \mathrm{sec}$ at $72^{\circ} \mathrm{C}$; and a final extension at $72^{\circ} \mathrm{C}$ for $5 \mathrm{~min}$. For analysis, $5 \mu \mathrm{l}$ of the product was subjected to agarose gel electrophoresis and visualized by ethidium bromide. PCR bands were quantified using the Multi Gauge V2.2 software program (FUJI PHOTO FILM, Japan).

Immunohistochemistry. Paraffin block was provided at Inje University Busan Paik Hospital (Busan, Korea). Immunohistochemical analysis was performed on $4-\mu \mathrm{m}$-thick paraffin-embedded sections of 42 tissues, which were mounted 
Table I. Primer sequences for RT-PCR analysis of PRDX.

\begin{tabular}{|c|c|c|}
\hline Gene & Primer sequence & Product size (bp) \\
\hline \multirow[t]{2}{*}{ PRDXI } & Forward 5'-GGGTATTCTTCGGCAGATCA-3' & 221 \\
\hline & Reverse 5'-GCAGCCTGGCACTAAAACAG-3' & \\
\hline \multirow[t]{2}{*}{$P R D X 2$} & Forward 5'-GTGTCCTTCGCCAGATCACT-3' & 154 \\
\hline & Reverse 5'-ACGTTGGGCTTAATCGTGTC-3' & \\
\hline \multirow[t]{2}{*}{$P R D X 3$} & Forward 5'-CAAGCAAAATTATTCAGCACCA-3' & 129 \\
\hline & Reverse 5'-CCCCTTAAAGTCATCAAGGCT-3' & \\
\hline \multirow[t]{2}{*}{$P R D X 4$} & Forward 5'-GAAATTATCGCTTTTGGCGA-3' & 149 \\
\hline & Reverse 5'-AGTGGAATCCTTATTGGCCC-3' & \\
\hline \multirow[t]{2}{*}{ PRDX5 } & Forward 5'-GTGGTGGCCTGTCTGAGTGT-3' & 150 \\
\hline & Reverse 5'-GGACACCAGCGAATCATCTA-3' & \\
\hline \multirow[t]{2}{*}{ PRDX6 } & Forward 5'-GGATGGGGATAGTGTGATGG-3' & 81 \\
\hline & Reverse 5'-TTGGTGAAGACTCCTTTCGG-3' & \\
\hline \multirow[t]{2}{*}{$h B 2 M F$} & Forward 5'-TGACTTTGTCACAGCCCAAG-3' & 265 \\
\hline & Reverse 5'-GAGCTACCTGTGGAGCAACC-3' & \\
\hline
\end{tabular}

PRDX, peroxiredoxin; hB2MF, human $\beta 2$-microglobulin.

on SuperFrost Plus slides. Slides were heated for $1 \mathrm{~h}$ at $60^{\circ} \mathrm{C}$, deparaffinized in xylene, rehydrated in graded alcohol and rinsed in distilled water. Antigen retrieval and immunohistochemistry were performed using either a Benchmark XT or Discovery XT automated immunohistochemistry system (Ventana Medical Systems, Inc., Tucson, AZ) with an OptiView DAB IHC Detection kit. Slides were incubated for $34 \mathrm{~min}$ at room temperature with diluted primary antibody. The appropriately diluted primary antibodies were as follows: PRDX1 (Abcam, Cambridge, MA, USA; cat. no. ab109506; dilution, 1:500), PRDX3 (Abcam; cat. no. ab16751; dilution, 1:1,000), PRDX5 (Abcam; cat. no. ab127922; dilution, 1:200), and PRDX6 (Abcam; cat. no. ab133348; dilution, 1:700). After antibody staining, sections were washed in distilled water, lightly counterstained with hematoxylin, rehydrated and mounted with coverslips. The intensity of immunohistochemical staining was evaluated in five randomly selected high-power fields using a light microscope (magnification $\mathrm{x} 400$ ). Sections were subsequently divided into four categories: -, no positive cells; + , weak cytoplasmic staining; ++, moderate cytoplasmic staining; +++ , strong cytoplasmic staining. The results were independently assessed by one investigator who was blinded to patient characteristics, stage and prognosis.

Statistical analysis. Statistical analyses were performed using MedCalc version 14.8.1 (Frank Schoonjans, Ghent University, Belgium). Categorical variables were compared using $\chi^{2}$ tests and Fisher's exact tests. The mean, median, and standard deviation were calculated for continuous variables and were compared using Mann-Whitney U-tests for two groups, Kruskal-Wallis tests for three or more unmatched groups, and Pearson's correlation coefficients. Survival analysis was performed by Kaplan-Meier analysis and generalized log-rank tests. $\mathrm{P}<0.05$ was considered to indicate a statistically significant difference.

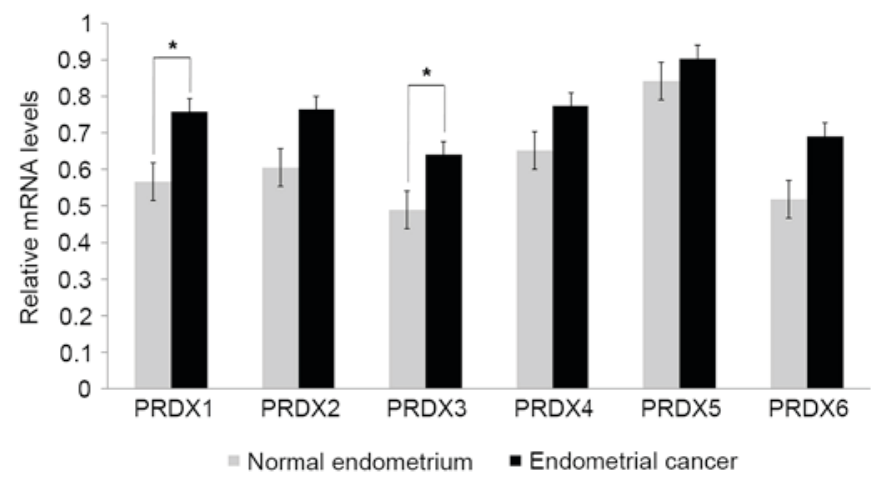

Figure 1.PRDX mRNA expression in endometrial cancer and normal endometrial tissues from benign uterine disease, as determined by semi-quantitative reverse transcription-polymerase chain reaction. All values represent the mean \pm standard deviation (bars) of three samples in independent experiments, which were repeated three times with similar results. ${ }^{*} \mathrm{P}<0.05$. PRDX, peroxiredoxin.

\section{Results}

Measurement of PRDX expression levels in endometrial cancer and normal endometrial tissues by RT-PCR. The expression levels of various PRDX mRNAs were analyzed in endometrial cancer $(n=26)$ and normal endometrial tissue $(n=10)$ to determine whether PRDX expression was associated with endometrial cancer. All PRDX mRNAs were upregulated in endometrial cancer compared with normal endometrial tissue (Fig. 1). Although the expression levels of PRDX2, PRDX4 and PRDX5 mRNAs were higher in endometrial cancer than in normal endometrium, these differences were not significant. Additionally, the expression level of PRDX6 mRNA in endometrial cancer was marginally higher than that in normal endometrium ( 0.52 vs. 0.69 , respectively; $\mathrm{P}=0.0612)$. Notably, the expression levels of PRDX1 (0.57 vs. 0.76, respectively; $\mathrm{P}=0.0015)$ and $\mathrm{PRDX} 3$ (0.49 vs. 0.64 , 
Table II. Intensity of immunostaining for PRDXs in normal endometrial tissue from cancer and endometrial cancer samples.

\begin{tabular}{|c|c|c|c|c|c|c|}
\hline \multirow[b]{2}{*}{ PRDX isoform } & \multirow[b]{2}{*}{ Sample type } & \multirow[b]{2}{*}{ No. of samples } & \multicolumn{3}{|c|}{ Staining intensity, n (\%) } & \multirow[b]{2}{*}{ P-value } \\
\hline & & & + & ++ & +++ & \\
\hline \multirow[t]{2}{*}{1} & Normal & 24 & $5(20.8)$ & $7(29.2)$ & $12(50.0)$ & NS \\
\hline & Cancer & 42 & $17(40.5)$ & $19(45.2)$ & $6(14.3)$ & \\
\hline \multirow[t]{2}{*}{3} & Normal & 19 & $3(15.8)$ & $10(52.6)$ & $6(31.6)$ & $0.0001^{\mathrm{a}}$ \\
\hline & Cancer & 42 & $6(14.3)$ & $11(26.2)$ & $25(59.5)$ & \\
\hline \multirow[t]{2}{*}{5} & Normal & 34 & $8(23.5)$ & $7(20.6)$ & $19(55.9)$ & $0.0023^{\mathrm{a}}$ \\
\hline & Cancer & 42 & $3(7.1)$ & 7 (16.7) & $32(76.2)$ & \\
\hline \multirow[t]{2}{*}{6} & Normal & 25 & $5(20.0)$ & $16(64.0)$ & $4(16.0)$ & NS \\
\hline & Cancer & 42 & $12(28.6)$ & $22(52.4)$ & $8(19.0)$ & \\
\hline
\end{tabular}

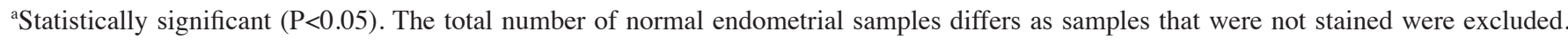
PRDX, peroxiredoxin; + , weak staining; ++ , moderate staining; +++ , strong staining; NS, no significance.
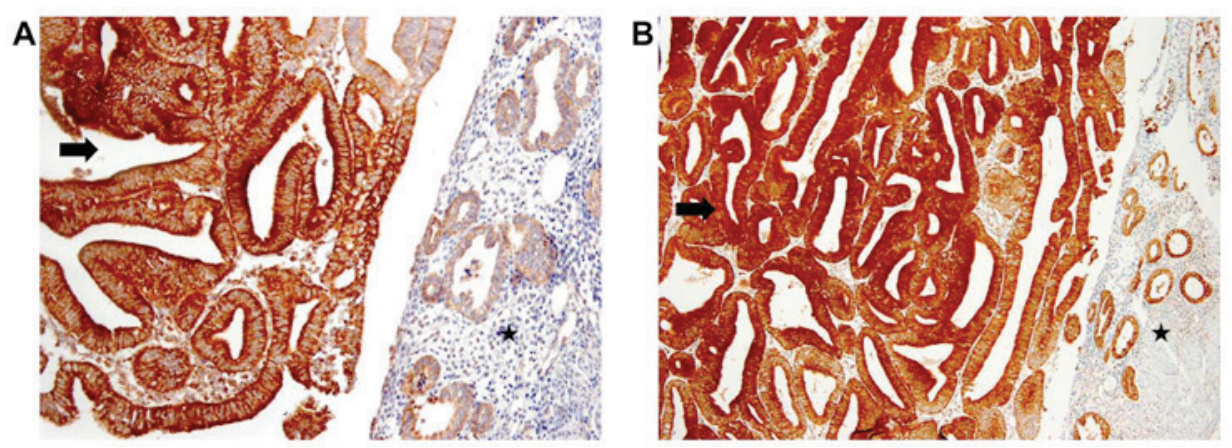

Figure 2. Immunohistochemical staining for (A) PRDX3 (magnification, x200) and (B) PRDX5 (magnification, x100) in endometrial cancer (arrow) and adjacent normal endometrial glands (star). PRDX, peroxiredoxin.
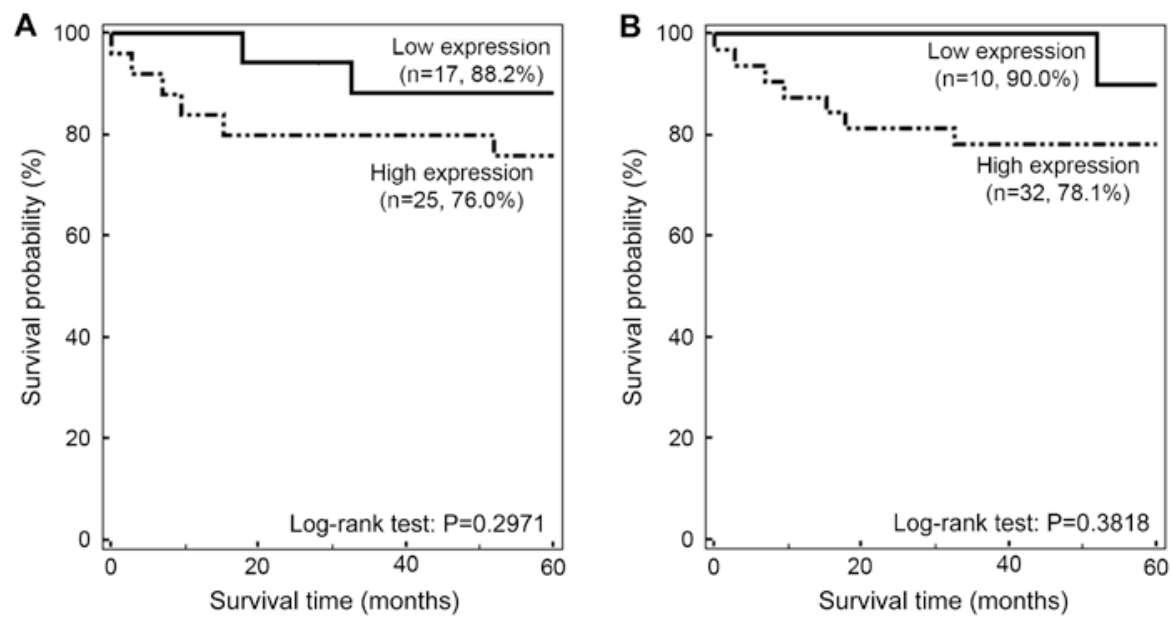

Figure 3. Survival rates of patients with endometrial cancer according to PRDX3 and PRDX5 expression levels. Kaplan-Meier survival curves showing cumulative survival rates of patients with (A) high or low PRDX3 expression, and (B) high or low PRDX5 expression. Percentages in brackets indicate the survival rate at 5 years. The results indicate that patients with endometrial cancer in the high PRDX3 and PRDX5 expression groups had a decreased 5-year survival rates compared with those in the low PRDX3 and PRDX5 expression groups. However, the associations between survival and PRDX expression were not statistically significant $(\mathrm{P}=0.2971$ and $\mathrm{P}=0.3818$, respectively). $\mathrm{PRDX}$, peroxiredoxin.

respectively; $\mathrm{P}=0.0134$ ) were significantly increased in endometrial cancer compared with those in normal endometrial tissues (Fig. 1).
Measurement of PRDX expression levels in endometrial cancer and paired normal endometrial tissues by immunohistochemistry. The expression levels of PRDX 


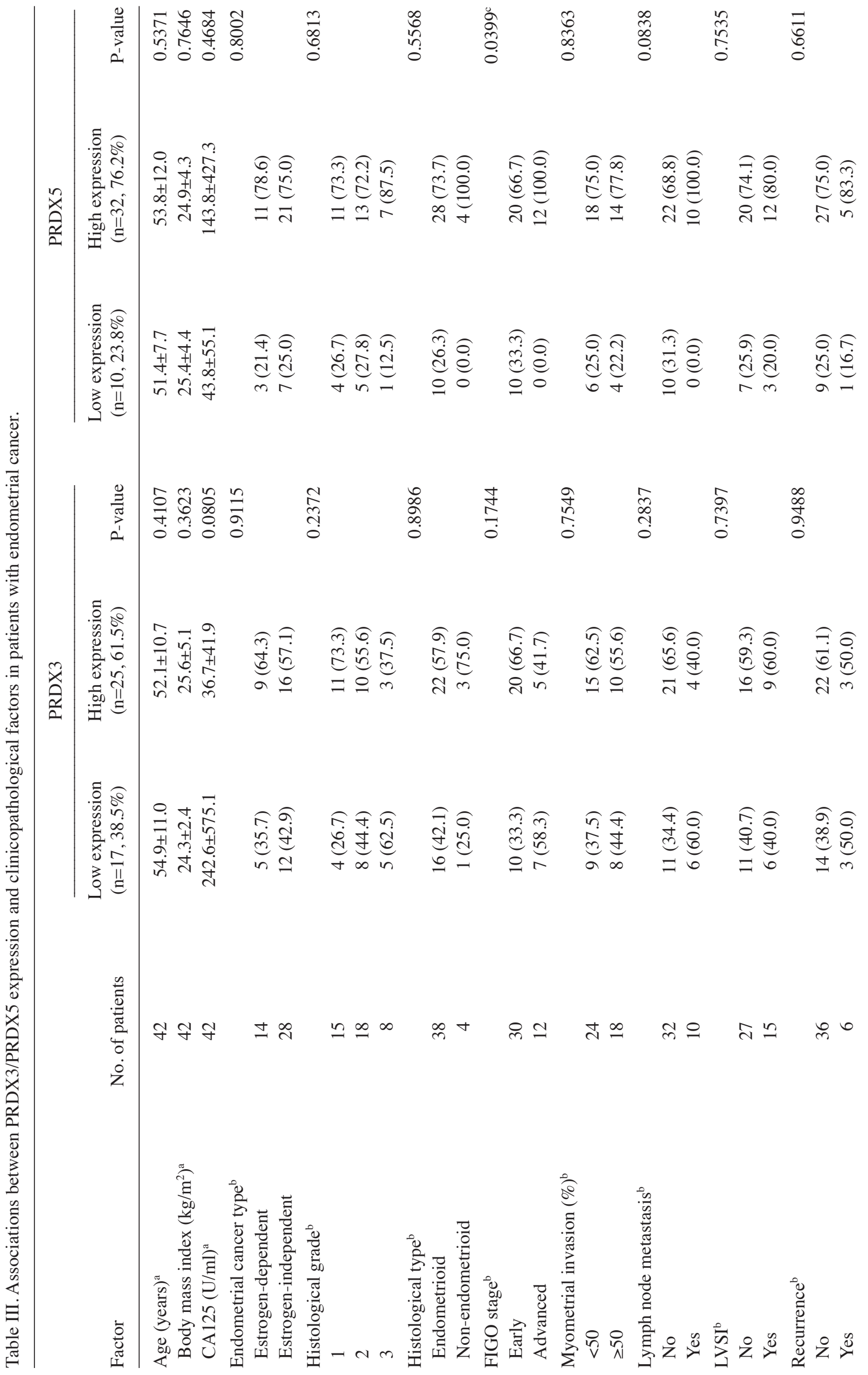


proteins were compared between endometrial cancer and paired normal endometrial tissues using immunohistochemistry. Based on the RT-PCR analysis, which detected marked changes in the expression of PRDX1, PRDX3, and PRDX6 mRNAs, and a previous study in which PRDX5 was found to be overexpressed in endometrial cancer (27), immunohistochemical staining for PRDX1, PRDX3, PRDX5, and PRDX6 was performed. The results showed that $59.5 \%$ of endometrial cancer samples and $31.6 \%$ of normal endometrial samples showed strong cytoplasmic staining for PRDX3, and statistical analysis revealed that PRDX3 was significantly overexpressed in endometrial cancer compared with normal endometrial tissue ( $\mathrm{P}=0.0001$; Table II; Fig. 2A). Similarly, a total $76.2 \%$ of endometrial cancer samples and $55.9 \%$ of normal endometrial tissues were strongly positive for PRDX5, and PRDX5 was significantly overexpressed in endometrial cancer compared with that in endometrial tissues ( $\mathrm{P}=0.0023$; Table II; Fig. 2B). However, no significant differences in the expression of PRDX 1 and 6 were observed between cancer and normal tissue.

Analysis of the association between PRDX3/PRDX5 overexpression and prognosis in patients with endometrial cancer. The associations between PRDX isoform expression and prognosis were evaluated in patients with endometrial cancer in order to investigate the usefulness of PRDX as a prognostic biomarker for endometrial cancer. As PRDX3 and PRDX5 were demonstrated to be overexpressed in endometrial cancer by immunohistochemistry, prognostic factors for endometrial cancer were investigated according to PRDX3 and PRDX5 expression. Patients were divided into the low (weak or moderate staining) and high (strong staining) PRDX3 and PRDX5 expression groups, and clinicopathological and prognostic variables of endometrial cancer, including grade, histology, FIGO stage, depth of myometrial invasion and lymph node status were compared between the low and high expression groups of each marker.

High PRDX5 expression was more frequently observed in advanced-stage cancer than in early-stage cancer (100 vs. 66.7\%; $\mathrm{P}=0.0399)$. Additionally, high expression of PRDX5 tended to be associated with the presence of lymph node metastasis; however, this association was not statistically significant (100 vs. 68.7\%; $\mathrm{P}=0.0838$ ). A total of 6 cases of recurrent cancer were reported, and all showed high expression of PRDX5. Additionally, 8 patients with endometrial cancer died during follow-up, and the majority of these patients exhibited high expression of PRDX3 (75\% vs. 55.9\% of patients who survived) and PRDX5 (87.5\% vs. $73.5 \%$ of patients who survived); however, no significant associations were detected (Table III).

Although the 5-year survival rate was increased in the low PRDX3 expression group compared with that in the high PRDX3 expression group (88.2 vs. $76.0 \%$, respectively), this association was not significant $(\mathrm{P}=0.2971$; Fig. 3A). Additionally, the 5-year survival rate in the low PRDX5 expression group was non-significantly higher than that in the high PRDX5 expression group (90 vs. $78.1 \%$; $\mathrm{P}=0.3818$; Fig. 3B).

\section{Discussion}

PRDX pathways are used by cells as enzymatic antioxidant defense systems in order to prevent oxidative and nitrosative 
damage caused by the presence of ROS (28). Among the six isoforms of PRDX (PRDX1-6), PRDX3 is localized in the mitochondria and acts as a mitochondrial scavenger of hydrogen peroxide, which protects mitochondria against oxidative damage and affects diverse cellular processes, including growth, differentiation, carcinogenesis and apoptosis (29). PRDX5 is localized in the cytosol, mitochondria, peroxisome and nucleus, and is able to reduce hydrogen peroxide, alkyl hydroperoxides, and peroxynitrite. PRDX5 is also able to use cytosolic and mitochondrial thioredoxins as physiological electron donors (30). These proteins are overexpressed in several types of malignancy, including breast cancer, mesothelioma, lung cancer, cervical cancer, prostate cancer, and multiple myeloma (31). Mitochondria in cancer cells are known to contain high levels of PRDX3 and PRDX5 (32-36), and Song et al (37) reported that the mitochondrial PRDX3 antioxidant system, which is exclusively present in mitochondria, may be a potential target for cancer therapy.

In the present study, the mRNA levels of all PRDX isoforms were higher in endometrial cancer than in normal endometrial tissue, with significant increases observed for $P R D X 1$ and PRDX3 mRNAs. However, on immunohistochemical analysis, PRDX3 and PRDX5 were clearly elevated in the majority of endometrial cancer samples, with PRDX3 protein showing significantly increased expression. Although PRDX1 mRNA expression was significantly higher in endometrial cancer, PRDX1 protein levels tended to be lower in endometrial cancer than in the normal endometrium. This difference between the mRNA and protein levels of PRDX1 may be due to the presence of extracellular PRDXs, such as macrophage PRDX or secreted PRDXs (38). As PRDX3 and PRDX5 were found to be highly expressed in endometrial cancer by RT-PCR and immunohistochemistry, these targets may be considered biomarkers associated with endometrial cancer.

Generally, factors associated with the prognosis of endometrial cancer include FIGO stage, histology, tumor grade, depth of myometrial invasion, lymphovascular space invasion, and lymph node status (39). In this study, high expression of PRDX5 was associated with advanced-stage endometrial cancer. Although not statistically significant, high expression of PRDX5 was also observed more frequently in patients with lymph node metastasis, and overexpression of PRDX3 and PRDX5 appeared more frequent in patients who died during follow-up.

The lack of significance among these findings could be explained by the small sample size and the limited stages and histological characteristics of the samples. Indeed, the majority of the samples were from patients with early-stage endometrial cancer and low-grade tumors. Therefore, more meaningful results may be obtained in studies with larger sample sizes and tumors with various clinicopathological characteristics.

In conclusion, PRDX3 and PRDX5 were highly expressed in endometrial cancer. In particular, the increased expression of PRDX5 was significantly associated with advanced stage, and tended to be increased among patients with positive lymph node status. Although no significant differences were detected in this analysis, the increased expression of PRDX3 and PRDX5 may be associated with decreased survival time. Therefore, these proteins may be candidate prognostic markers in patients with endometrial cancer. Additional studies with larger sample sizes are required to fully determine the prognostic roles of PRDX3 and PRDX5 in endometrial cancer.

\section{Competing interests}

The authors declare that they have no competing interests.

\section{References}

1. Ferlay J, Soerjomataram I, Dikshit R, Eser S, Mathers C, Rebelo M, Parkin DM, Forman D and Bray F: Cancer incidence and mortality worldwide: Sources, methods and major patterns in GLOBOCAN 2012. Int J Cancer 136: E359-E386, 2015.

2. Evans T, Sany O, Pearmain P, Ganesan R, Blann A and Sundar S: Differential trends in the rising incidence of endometrial cancer by type: Data from a UK population-based registry from 1994 to 2006. Br J Cancer 104: 1505-1510, 2011.

3. Fei LY: Fast Stats: An interactive tool for access to SEER cancer statistics. Surveillance Research Program, National Cancer Institute. http://seer.cancer.gov/faststats. (Accessed on February 2, 2013.

4. Smith-Bindman R, Kerlikowske K, Feldstein VA, Subak L, Scheidler J, Segal M, Brand R and Grady D: Endovaginal ultrasound to exclude endometrial cancer and other endometrial abnormalities. JAMA 280: 1510-1517, 1998

5. Committee on Practice Bulletins-Gynecology: Practice bulletin no. 128: Diagnosis of abnormal uterine bleeding in reproductive-aged women. Obstet Gynecol 120: 197-206, 2012.

6. Berek JS: Novak's Gynecology 13th edition. Lippincott Williams \& Wilkins, Philadelphia, PA, USA, 2002.

7. Ueda Y, Enomoto T, Kimura T, Miyatake T, Yoshino K, Fujita M and Kimura T: Serum biomarkers for early detection of gynecologic cancers. Cancers (Basel) 2: 1312-1327, 2010.

8. Gadducci A, Cosio S and Genazzani AR: Tissue and serum biomarkers as prognostic variables in endometrioid-type endometrial cancer. Crit Rev Oncol Hematol 80: 181-192, 2011.

9. Hakala A, Kacinski BM, Stanley ER, Kohorn EI, Puistola U, Risteli J, Risteli L, Tomás C and Kauppila A: Macrophage colony-stimulating factor 1 , a clinically useful tumor marker in endometrial adenocarcinoma: comparison with CA 125 and the aminoterminal propeptide of type III procollagen. Am J Obstet Gynecol 173: 112-119, 1995.

10. Takeshima N, Shimizu Y, Umezawa S, Hirai Y, Chen JT, Fujimoto I, Yamauchi K and Hasumi K: Combined assay of serum levels of CA125 and CA19-9 in endometrial carcinoma. Gynecol Oncol 54: 321-326, 1994.

11. Baser E, Gungor T, Togrul C, Turkoglu O and Celen S: Preoperative prediction of poor prognostic parameters and adjuvant treatment in women with pure endometrioid type endometrial cancer: What is the significance of tumor markers? Eur J Gynaecol Oncol 35: 513-518, 2014.

12. Yasa C, Takmaz O, Dural O and Akhan SE: The value of tumor markers in endometrial carcinoma: Review of literature. Sci Res 4: 966-970, 2013.

13. Price FV, Chambers SK, Carcangiu ML, Kohorn EI, Schwartz PE and Chambers JT: CA 125 may not reflect disease status in patients with uterine serous carcinoma. Cancer 82: 1720-1725, 1998.

14. Kim K, Kim I, Lee KY, Rhee S and Stadtman E: The isolation and purification of a specific 'protector' protein which inhibits enzyme inactivation by a thiol/Fe (III)/O2 mixed-function oxidation system. J Biol Chem 263: 4704-4711, 1988.

15. Rhee SG: Cell signaling. H2O2, a necessary evil for cell signaling. Scienc e 312: 1882-1883, 2006.

16. Hall A, Nelson K, Poole LB and Karplus PA: Structure-based insights into the catalytic power and conformational dexterity of peroxiredoxins. Antioxid Redox Signal 15: 795-815, 2011.

17. Neumann CA, Cao J and Manevich Y: Peroxiredoxin 1 and its role in cell signaling. Cell Cycle 8: 4072-4078, 2009.

18. Mu ZM, Yin XY and Prochownik EV: Pag, a putative tumor suppressor, interacts with the Myc Box II domain of c-Myc and selectively alters its biological function and target gene expression. J Biol Chem 277: 43175-43184, 2002.

19. Yo YD, Chung YM, Park JK, Ahn CM, Kim SK and Kim HJ: Synergistic effect of peroxiredoxin II antisense on cisplatin-induced cell death. Exp Mol Med 34: 273-277, 2002. 
20. Okoh V, Deoraj A and Roy D: Estrogen-induced reactive oxygen species-mediated signalings contribute to breast cancer. Biochim Biophys Acta 1815: 115-133, 2011.

21. Felty Q, Xiong WC, Sun D, Sarkar S, Singh KP, Parkash J and Roy D: Estrogen-induced mitochondrial reactive oxygen species as signal-transducing messengers. Biochemistry 44: 6900-6909, 2005.

22. Musarrat J, Arezina-Wilson J and Wani A: Prognostic and aetiological relevance of 8-hydroxyguanosine in human breast carcinogenesis. Eur J Cancer 32A: 1209-1214, 1996.

23. O'Leary PC, Terrile M, Bajor M, Gaj P, Hennessy BT, Mills GB, Zagozdzon A, O'Connor DP, Brennan DJ, Connor K, et al: Peroxiredoxin-1 protects estrogen receptor alpha from oxidative stress-induced suppression and is a protein biomarker of favorable prognosis in breast cancer. Breast Cancer Res 16: R79, 2014.

24. Karihtala P, Kauppila S, Soini Y and Arja-Jukkola-Vuorinen: Oxidative stress and counteracting mechanisms in hormone receptor positive, triple-negative and basal-like breast carcinomas. BMC Cancer 11: 262, 2011.

25. Elamin A, Zhu H, Hassan AM, Xu N and Ibrahim ME: Peroxiredoxin V: A candidate breast tumor marker of population specificity. Mol Clin Oncol 1: 541-549, 2013.

26. Pecorelli S: Revised FIGO staging for carcinoma of the vulva, cervix, and endometrium. Int J Gynaecol Obstet 105: 103-104, 2009.

27. Han S, Shen H, Jung M, Hahn BS, Jin BK, Kang I, Ha J and Choe W: Expression and prognostic significance of human peroxiredoxin isoforms in endometrial cancer. Oncol Lett 3: 1275-1279, 2012.

28. Rhee SG, Chae HZ and Kim K: Peroxiredoxins: A historical overview and speculative preview of novel mechanisms and emerging concepts in cell signaling. Free Radic Biol Med 38: 1543-1552, 2005

29. Wonsey DR, Zeller KI and Dang CV: The c-Myc target gene PRDX3 is required for mitochondrial homeostasis and neoplastic transformation. Proc Natl Acad Sci USA 99: 6649-6654, 2002.

30. Knoops B, Clippe A, Bogard C, Arsalane K, Wattiez R, Hermans C, Duconseille E, Falmagne $\mathrm{P}$ and Bernard A: Cloning and characterization of AOEB166, a novel mammalian antioxidant enzyme of the peroxiredoxin family. J Biol Chem 274: 30451-30458, 1999.
31. Whitaker HC, Patel D, Howat WJ, Warren AY, Kay JD, Sangan T, Marioni JC, Mitchell J, Aldridge S, Luxton HJ, et al: Peroxiredoxin-3 is overexpressed in prostate cancer and promotes cancer cell survival by protecting cells from oxidative stress. Br J Cancer 109: 983-993, 2013.

32. Kinnula VL, Lehtonen S, Sormunen R, Kaarteenaho-Wiik R, Kang SW, Rhee SG and Soini Y: Overexpression of peroxiredoxins I, II, III, V, and VI in malignant mesothelioma. J Pathol 196: 316-323, 2002.

33. Noh DY, Ahn SJ, Lee RA, Kim SW, Park IA and Chae HZ: Overexpression of peroxiredoxin in human breast cancer. Anticancer Res 21: 2085-2090, 2001.

34. Choi JH, Kim TN, Kim S, Baek SH, Kim JH, Lee SR and Kim JR: Overexpression of mitochondrial thioredoxin reductase and peroxiredoxin III in hepatocellular carcinomas. Anticancer Res 22: 3331-3335, 2002.

35. Nonn L, Berggren M and Powis G: Increased expression of mitochondrial peroxiredoxin-3 (Thioredoxin Peroxidase-2) protects cancer cells against hypoxia and drug-induced hydrogen peroxide-dependent. Mol Cancer Res 1: 682-689, 2003.

36. Kropotov A, Gogvadze V, Shupliakov O, Tomilin N, Serikov VB, Tomilin NV and Zhivotovsky B: Peroxiredoxin V is essential for protection against apoptosis in human lung carcinoma cells. Exp Cell Res 312: 2806-2815, 2006.

37. Song IS, Kim HK, Jeong SH, Lee SR, Kim N, Rhee BD, Ko KS and Han J: Mitochondrial peroxiredoxin III is a potential target for cancer therapy. Int J Mol Sci 12: 7163-7185, 2011.

38. Riddell JR, Wang XY, Minderman H and Gollnick SO: Peroxiredoxin 1 stimulates secretion of proinflammatory cytokines by binding to TLR4. J Immunol 184: 1022-1030, 2010.

39. Creasman WT, Odicino F, Maisonneuve P, Quinn MA, Beller U, Benedet JL, Heintz AP, Ngan HY and Pecorelli S: Carcinoma of the corpus uteri. FIGO 26th annual report on the results of treatment in gynecological cancer. Int J Gynaecol Obstet 95 (Suppl 1): S105-S143, 2006. 